\title{
On-Chip, Ultra-Low Threshold Yb Silica Laser
}

\author{
Eric P. Ostby, Lan Yang, and Kerry J. Vahala \\ California Institute of Technology, 1200 E. Colorado Blvd., Pasadena, CA 91125 \\ ostby@caltech.edu http://www.vahala.caltech.edu
}

\begin{abstract}
A novel $\mathrm{Yb}: \mathrm{SiO}_{2}$ fiber-coupled laser on a silicon chip was fabricated using a solution-gel process. We report a record-low pump threshold of $2 \mu \mathrm{W}$, and discuss the practical advantages of $\mathrm{Yb}$ microlasers.

(C) 2007 Optical Society of America

OCIS: (140.3070) Infrared and far-infrared lasers; (140.5680) Rare earth and transition metal solid-state lasers
\end{abstract}

\section{Introduction}

There is great interest in silicon-chip-based sources for various applications including sensing and communications. The high cavity quality factor $(\mathrm{Q})$ of a silica microtoroid fabricated on a silicon chip enables low pump threshold for Raman, parametric, or rare-earth-doped laser operation [1,2]. The sol-gel method we use to construct silica from a metal alkoxide precursor provides a simple and flexible way to tailor the dopants of the laser gain medium. Using this technique, our group has already demonstrated an erbium doped silica (Er: $\left.\mathrm{SiO}_{2}\right)$ microtoroid laser in the optical communication window at $1.55 \mu \mathrm{m}$ [3]. But, ytterbium has several advantages including no up-conversion or concentration-quenching (due to its simple electronic structure), which limit the doping to less than $1 \%$ for erbium. Also, the pump absorption crosssection of $\mathrm{Yb}$ doped silica $\left(\mathrm{Yb}: \mathrm{SiO}_{2}\right)$ is $27 \times 10^{-21} \mathrm{~cm}^{-3}$, more than an order of magnitude higher compared to erbium. Finally, the higher gain possible in $\mathrm{Yb}: \mathrm{SiO}_{2}$ reduces the minimum cavity $\mathrm{Q}$ factor necessary for lasing, making fabrication more practical.

\section{Fabrication}

The microtoroid laser cavity is progressively built-up using chemistry, lithography, and annealing. First, we hydrolyze the tetraethoxysilane (TEOS) with water and then add hydrochloric acid. $\mathrm{Yb}^{3+}$ ions are incorporated by mixing in the correct amount of ytterbium nitrate to yield the desired concentration in glass. This $\mathrm{Yb}: \mathrm{SiO}_{2}$ has a $\mathrm{Yb}^{3+}$ concentration of $2 \times 10^{19} \mathrm{~cm}^{-3}$. The chemical reaction then continues for 3 hours at $70^{\circ} \mathrm{C}$ on a hot plate. At this point, the viscous solution is spin-coated on a silicon wafer and annealed at $1,000^{\circ} \mathrm{C}$ for 3 hours to remove solvents and complete the transition to amorphous glass. Three consecutive spin and anneal steps produce a $1.5 \mu \mathrm{m}$ thin film of $\mathrm{Yb}: \mathrm{SiO}_{2}$. Standard optical lithography and buffered $\mathrm{HF}$ etch are used to create $80 \mu \mathrm{m}$ silica disks. To prevent optical leakage to the silicon, $\mathrm{XeF}_{2}$ dry etch creates an array of silica disks supported underneath by silicon pillars. As a final step, the rough silica disks are reflowed into smooth toroids by $\mathrm{CO}_{2}$ laser annealing as described elsewhere [1]. Figure 1 shows side and top views of the $\mathrm{Yb}: \mathrm{SiO}_{2}$ microcavity discussed in this paper.

Fig. 1. Side and top views of $\mathrm{Yb}: \mathrm{SiO}_{2}$ microcavity
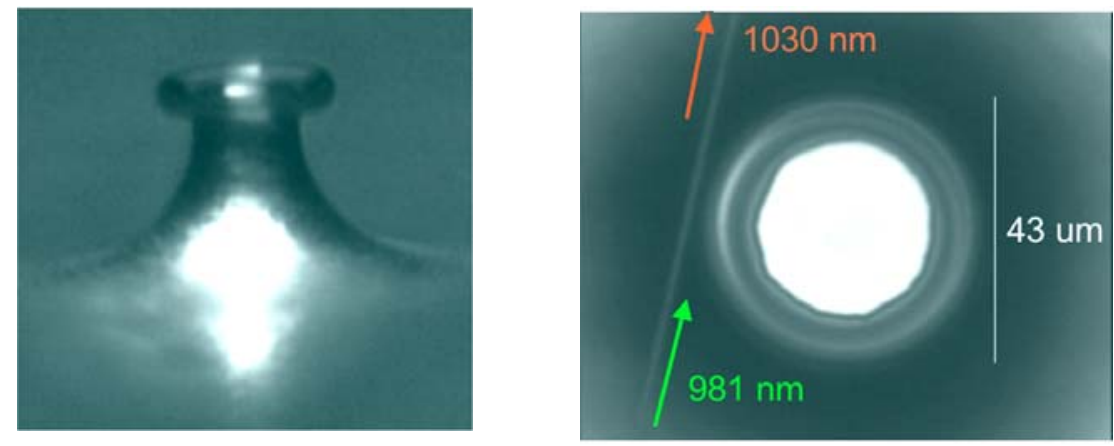


\section{CFJ1.pdf}

\section{Laser Testing}

Laser pump light $(970 \mathrm{~nm})$ from a single-frequency diode laser is fiber-coupled to the microcavity by means of a phase-matched fiber taper as shown in Figure 1. Laser emission $(1030-1060 \mathrm{~nm})$ is efficiently coupled out of the cavity using the same fiber. A WDM filter is used to separate the pump and signal light. The air-gap between the fiber taper and toroid is adjusted for optimal coupling using nanocube stages. Numerous cavity resonances in this 43 um diameter $\mathrm{Yb}: \mathrm{SiO}_{2}$ were investigated to find the optimum pump wavelength. The broad pump and emission bandwidths of $\mathrm{Yb}$ permit laser output from $965-985 \mathrm{~nm}$. The best laser performance is at $970.2 \mathrm{~nm}$. Since optical Q depends greatly on loss (absorption), the optical Q at $970 \mathrm{~nm}$ is only $3.7 \times 10^{5}$. Far from the absorption line, the $\mathrm{Q}$ increases to $5.5 \times 10^{6}$ at $1550 \mathrm{~nm}$. Since this microcavity lases easily with a relatively low $\mathrm{Q}$, one can see that $\mathrm{Yb}$ enables low-pump-power on-chip lasers with more practical and easily attainable quality factors.

Figure $\mathbf{2}$ is a plot of the output power as a function of the absorbed pump power. The linear fit shows that this $\mathrm{Yb}: \mathrm{SiO}_{2}$ microlaser has a low pump threshold of only $2 \mu \mathrm{W}$, which is $3,000 \mathrm{x}$ smaller than the lowest published value to date for any $\mathrm{Yb}$ laser [4]. The laser slope efficiency is $11 \%$, and the output power is greater than $22 \mu \mathrm{W}$ and limited only by the $1 \mathrm{~mW}$ available pump power.

Fig. 2. Output power vs. pump power

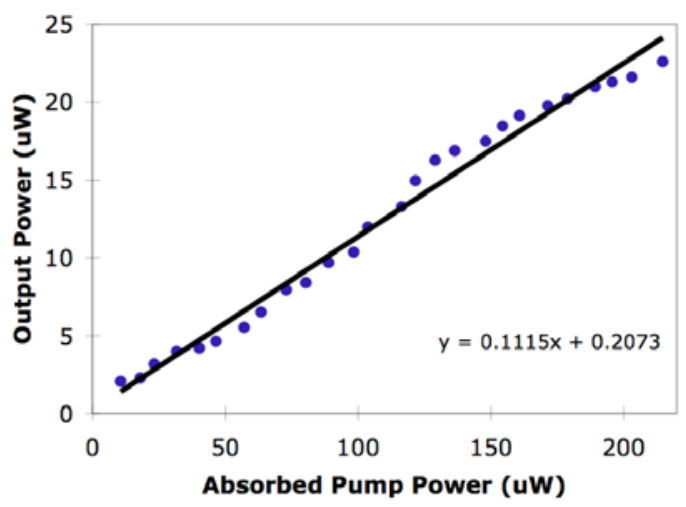

Fig. 3. Optical spectrum

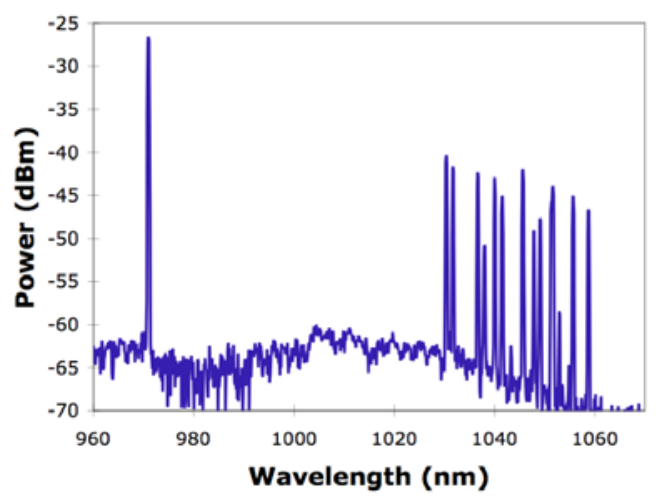

The laser emits infrared radiation in multiple modes as demonstrated in the optical spectrum in Figure 3. The emission spectrum spans $1030-1060 \mathrm{~nm}$, and is not unexpected given $\mathrm{Yb}$ 's broad emission bandwidth. Careful control of the toroid's minor diameter and taper coupling may enable single mode lasing.

\section{Future Work}

$\mathrm{Yb}: \mathrm{SiO}_{2}$ microcavity lasers may have applications in on-chip optical communication, biological or material sensing, and other devices that require an efficient and low power laser. In the future, we will incorporate other ions into the silica during the sol-gel fabrication to improve laser efficiency. We are also interested in a high peak power and pulsed on-chip laser that can be used for broad-spectrum non-linear studies.

In conclusion, we have presented a sol-gel fabricated $\mathrm{Yb}: \mathrm{SiO}_{2}$ microlaser with a record low $2 \mu \mathrm{W}$ pump threshold. The robust laser performance and fabrication flexibility of this on-chip laser offer many opportunities for future investigations.

\section{References}

[1] D. K. Armani et al., "Ultra-high-Q toroid microcavity on a chip", Nature, 421, 925-929 (2003).

[2] T. J. Kippenberg et al., "Ultralow-threshold microcavity Raman laser on a microelectronic chip”, Opt. Lett., 29, 1224-1227 (2004).

[3] Lan Yang et al., "Erbium-doped and Raman microlasers on a silicon chip fabricated by the sol-gel process", Appl. Phys. Lett. 86, 091114 (2005).

[4] Pask et al., "Ytterbium-doped silica fiber lasers: versatile sources for the 1-1.2 $\mu \mathrm{m}$ region", IEEE J. Quant. Elect. 1, 2-13 (1995). 